\title{
Neutrophil extracellular traps and heparin-induced antibodies contribute to vascular access thrombosis in hemodialysis patients
}

\author{
Hoi Woul Lee ${ }^{1}$, Jung Nam $\mathrm{An}^{2}$, Hyung Seok Lee ${ }^{2}$, Young Rim Song ${ }^{2}$, Hyung Jik Kim², Sung Gyun Kim ${ }^{1,2}$, \\ Jwa-Kyung Kim ${ }^{1,2}$ \\ ${ }^{1}$ Department of Clinical Immunology, Hallym University Sacred Heart Hospital, Anyang, Republic of Korea \\ ${ }^{2}$ Department of Internal Medicine and Kidney Research Institute, Hallym University Sacred Heart Hospital, Anyang, Republic of Korea
}

Background: Anti-heparin/platelet factor 4 (PF4) antibodies may trigger severe thrombotic complications in hemodialysis (HD) patients. Tetrameric PF4 has a high affinity for extracellular DNA, which is a key component of neutrophil extracellular traps (NETs); therefore, the interactions between anti-heparin/PF4 antibodies and NETs can contribute to prothrombotic events.

Methods: Anti-heparin/PF4 antibody levels were measured by enzyme-linked immunosorbent assay and an optical density $>1.8$ was regarded as clinically significant. We additionally measured serum nucleosome levels as representative markers of NETs, and the contributions of anti-heparin/PF4 and increased serum nucleosome levels to the primary functional patency loss of vascular access was assessed.

Results: The frequency of anti-heparin/PF4 antibodies was significantly higher in incident HD patients compared to prevalent HD patients (23.6\% vs. $7.7 \%$ ). Serum nucleosome levels, as well as the white blood cell counts, neutrophil counts, and high- sensitivity C-reactive protein levels, were significantly higher in anti-heparin/PF4 antibody-positive patients compared to the control. Platelet counts tended to be lower in the patients with anti-heparin/PF4 of $>1.8$ than in the controls. Relative risk calculations showed that the presence of anti-heparin/PF4 antibodies increased the risk of primary functional patency failure by 4.28 -fold, and this risk increased further with higher nucleosome levels. Furthermore, in the anti-heparin/PF4 antibody-positive group, the time to first vascular intervention was much shorter, and the risk of repeated intervention was higher, compared to the controls.

Conclusion: In incident HD patients, the presence of anti-heparin/PF4 antibodies was associated with increased NET formation; this could be a strong predictor of vascular access complications

Keywords: Extracellular traps, Platelet factor 4, Renal dialysis, Thrombosis, Vascular access

Received: April 12, 2021; Revised: May 10, 2021; Accepted: June 3, 2021

Editor: Soon Hyo Kwon, Soonchunhyang University, Seoul, Republic of Korea

Correspondence: Jwa-Kyung Kim

Department of Internal Medicine and Kidney Research Institute, Hallym University Sacred Heart Hospital, 22 Gwanpyeong-ro 170beon-gil,

Dongan-gu, Anyang 14068, Republic of Korea. E-mail: kjk816@hallym.or.kr

ORCID: https://orcid.org/0000-0002-7726-2143

\section{Sung Gyun Kim}

Department of Internal Medicine and Kidney Research Institute, Hallym University Sacred Heart Hospital, 22 Gwanpyeong-ro 170beon-gil,

Dongan-gu, Anyang 14068, Republic of Korea. E-mail: imnksk@gmail.com

ORCID: https://orcid.org/0000-0002-5034-0527

Jwa-Kyung Kim and Sung Gyun Kim contributed equally to this study.

Copyright (C) 2021 by The Korean Society of Nephrology

(a) This is an Open Access article distributed under the terms of the Creative Commons Attribution Non-Commercial and No Derivatives License (http:// creativecommons.org/licenses/by-nc-nd/4.0/) which permits unrestricted non-commercial use, distribution of the material without any modifications, and reproduction in any medium, provided the original works properly cited. 


\section{Introduction}

Heparin-induced thrombocytopenia (HIT) is a serious immune reaction to heparin often characterized by severe thrombosis; the morbidity and mortality rates of HIT patients are high [1,2]. HIT is caused by the formation of a complex of abnormal autoantibodies that bind to platelet factor 4 (PF4) and heparin, together known as HIT antibodies [3]. HIT antibodies activate platelets, which can cause catastrophic arterial and venous thrombosis. HIT occurs in approximately $5 \%$ of patients exposed to heparin, regardless of the dose, schedule, or route of administration [4-6].

Patients undergoing continuous renal replacement therapy or intermittent hemodialysis (HD) typically require anticoagulation treatment to prevent the clotting of extracorporeal circuits. Heparin is the drug most commonly used for this purpose [7]. Although long-term heparin exposure is associated with a high incidence of anti-heparin antibodies, the prevalence and clinical significance of HIT antibody formation are currently unknown [8-10]. A previous study examining 700 incident $\mathrm{HD}$ and peritoneal dialysis patients reported a relatively high incidence of anti-heparin/PF4 antibodies [8]. However, they found no correlation between the presence of anti-heparin/PF4 and thrombocytopenia or thrombosis, and their study did not predict clinical outcomes. As a result, routine testing for the presence of HIT antibodies is not currently recommended for HD patients $[8,11,12]$. However, the mortality rate of untreated HIT has been reported to be as high as $20 \%$ in HD patients [13-15].

There are several phases of HIT, including an acute phase and various recovery phases [16]. Subclinical HIT, or subacute HIT, refers to the state in which a patient has recovered from an acute episode of HIT, but has persistent HIT antibodies; in this case, the presence of HIT antibodies may not be associated with clinical outcomes [17]. Furthermore, some patients develop clinically silent or insignificant anti-PF4 antibodies, which do not cause thrombocytopenia. Therefore, the timing of the detection and evaluation of anti-heparin/PF4 antibodies may determine their prognostic significance [6].

Unlike most other forms of drug-induced immune thrombocytopenia, HIT is associated with vascular thrombosis, with the primary mechanism of platelet activation and resultant injury to endothelial cells [18]. Recently, neutrophil extracellular traps (NETs), or NETosis, has been implicated in the development of thrombosis in individuals with HIT [6,19-21]. HIT immune complexes can activate neutrophils either directly, via the Fc-gamma receptor IIA (FcrRIIA), or indirectly, via P-selectin and its ligand P-selectin glycoprotein ligand-1. Furthermore, PF4 adheres readily to NET DNA, and the resultant PF4-NET complex contributes to the prothrombotic nature of HIT $[19,20,22]$. A mouse model study of HIT revealed that both the depletion of neutrophils and the blockage of neutrophil nuclear decondensation caused by a NETosis inhibitor prevented thrombosis, even though thrombocytopenia was unaffected, thus demonstrating the critical role of NETosis in HIT thrombosis [19].

Given that vascular access complications are largely due to the thrombotic occlusion of a stenotic fistula or graft [23,24], we assessed whether anti-heparin/PF4 antibodies played a role in adverse HD vascular access outcomes. We compared the prevalence of heparin-induced anti-heparin/PF4 antibodies between incident HD patients, who had recently been exposed to heparin for the first time, and maintenance HD (MHD) patients, who had been undergoing regular dialysis for more than 3 months with long-term heparin use. As a negative control, we also measured anti-heparin/PF4 antibodies in HD patients who did not use heparin. Furthermore, we measured serum nucleosome levels as a representative marker of NET in vivo. In this manner, we evaluated the utility of the association between anti-heparin/PF4 antibodies and nucleosome levels for predicting adverse HD vascular access outcomes in incident $\mathrm{HD}$ patients.

\section{Methods}

\section{Study population}

This observational, single-center study included incident HD patients, who had recently started dialysis treatment, and MHD patients, who underwent regular HD (three times per week), between January 2016 and June 2017. Of the 105 incident HD patients, 31 were excluded for the following reasons; presence of an acute infection at the time of starting HD ( $\mathrm{n}=3)$, decompensated liver cirrhosis $(\mathrm{n}=2)$, decompensated heart failure with ejection fraction of $<30 \%(n=6)$, hematologic diseases $(n=1)$, nonachievement of long-term vascular access $(n=10)$, death or recovery of renal funcion within 3 months $(n=3)$, or loss to follow up or transfer to another clinic $(n=6)$. Of the 169 MHD patients, 26 were 
excluded due to the presence of hematologic diseases ( $\mathrm{n}$ $=3)$, decompensated liver cirrhosis $(\mathrm{n}=2)$, decompensated heart failure with ejection fraction of $<30 \%(n=4)$, nonachievement of long-term vascular access $(n=12)$, or nonuse of heparin due to increased bleeding tendency ( $\mathrm{n}=$ 5). In total, 74 incident HD patients and 143 MHD patients were analyzed. Among the 74 incident HD patients, 55 used unfractionated heparin for anticoagulation, and 19 did not use heparin due to recent gastrointestinal bleeding $(n=5)$, a large hematoma or bruise associated with vascular access $(\mathrm{n}=4)$, or some other reason $(\mathrm{n}=10)$. The study protocol was approved by the Institutional Review Board of Hallym University Sacred Heart Hospital (No. 2016-I067), and informed consent was obtained from all patients.

\section{Data collection and blood sampling}

Baseline demographic data were obtained, including age, sex, and comorbidities, along with clinical data regarding the underlying cause of renal disease. Venous blood samples were collected into K2-ethylenediaminetetraacetic acid (EDTA)-coated tubes and 0.109 M trisodium citrate-coated tubes (Becton Dickinson, Franklin Lakes, NJ, USA). For the isolation of plasma, the blood was separated by centrifugation at $1,500 \times g$ for 20 minutes at $4^{\circ} \mathrm{C}$. Isolated plasma samples were aliquoted and stored at $-80^{\circ} \mathrm{C}$ until analysis.

For MHD patients, sampling was performed immediately before the HD session in the middle of the week. Biochemical analyses of white blood cells (WBCs), neutrophils, lymphocytes, platelets, hemoglobin, serum albumin, cholesterol, blood urea nitrogen, and creatinine were performed. The neutrophil to lymphocyte ratio was also calculated. Levels of the high-sensitivity C-reactive protein (hs-CRP) inflammatory cytokine were also measured.

\section{Anti-heparin/platelet factor 4 antibody and neutrophil extracellular trap quantification assays}

The anti-heparin/PF4 antibodies were detected using a commercial heparin/PF4 enzyme-linked immunosorbent assay (ELISA) kit (PF4 IgG, HAT 45G; Immucor GTI Diagnostics, Waukesha, WI, USA) [17]. Diluted, citrated plasma (1:50) was added to microwells, which were coated with immobilized PF4 complexed to polyvinyl sulfonate. After incubation for 35 minutes in a $37^{\circ} \mathrm{C}$ water bath, the microwells were washed and incubated with alkaline phosphatase-conjugated anti-human immunoglobulin (IgG) antibody. Following a second incubation for 35 minutes in a $37^{\circ} \mathrm{C}$ water bath, the microwells were washed and incubated with p-nitrophenyl phosphate substrate for 30 minutes. Optical density (OD) was measured by subtracting the absorbance reading at 490 $\mathrm{nm}$ (a reference filter) from the absorbance reading at 405 $\mathrm{nm}$. According to a previous study, OD values of $<0.4$ indicated an absence of antibodies. OD values equal to or greater than 1.0 indicated the presence of antibodies; values higher than 1.8 were regarded as clinically significant (strongly positive). The differences between the duplicate wells were $<20 \%[11,25]$.

To evaluate NET formation, histone-DNA complex (nucleosome) NET biomarkers were measured using a Cell Death Detection ELISA ${ }^{\text {PLUS }}$ Kit (Roche, Mannheim, Germany). According to the manufacturer's instructions, a mixture containing anti-histone-biotin antibody, anti-DNA-peroxidase antibody, and EDTA-plasma was placed in streptavidin-coated wells. After incubation for 2 hours, the wells were washed and reacted with a substrate for 20 minutes. The reaction was stopped using a stop solution. OD was calculated in the same way as in the heparin-PF4-IgG antibody assay. As we reported previously, the highest quartile of serum nucleosome (Q4) was used as the cutoff value for significant increase of NETs [26].

\section{Study endpoints}

The primary outcomes of this study were primary functional patency loss, defined as thrombotic occlusion of vascular access (including abandonment of the access site) requiring surgical or percutaneous endovascular intervention (percutaneous transluminal angioplasty [PTA] or thrombectomy) following initial cannulation, and achievement of adequate dialysis within 6 months of the first dialysis session $[1,27]$.

The secondary outcomes were as follows; the time taken for successful cannulation in three consecutive HD sessions, the time to the first intervention and frequency of first interventions within 3 months, and the time between the first intervention and reintervention. We also analyzed the abandonment rate of initial vascular access during follow-up. 


\section{Statistical analysis}

As an exploratory study, the sample size of the study was planned based on the expected mean differences between the incident HD, MHD, and control populations (non-heparin users) of $20 \%$ of the anti-PF4 antibody level. Using nonparametric sample size estimation, the sample size calculation for the incident HD patients resulted in a sample size of 50 to 60 patients and control groups who were eligible for the study in the same study period to achieve a power of at least $80 \%$. Variables with normal distributions are expressed as the means \pm standard deviations, and the Kolmogorov-Smirnov test was used to analyze the normality of the distribution of each parameter. Categorical variables are expressed as percentages and were compared using the chi-square test. Cumulative survival curves were derived using the Kaplan-Meier method, and differences between survival curves were compared using the log-rank test. Pearson's correlation analysis was used to clarify the relation ship between the number of immune cells and anti-PF4 antibody levels. A Cox proportional hazards model was used to iden- tify factors independently associated with vascular access abandonment, based on hazard ratios (HRs) with 95\% confidence intervals (CIs). A p-value less than 0.05 was regarded as significant. All statistical analyses were performed using IBM SPSS version 22.0 (IBM Corp., Armonk, NY, USA).

\section{Results}

\section{Baseline characteristics}

During the 18-month period from January 2016 to June 2017, a total of 105 incident HD and 169 MHD patients were enrolled in this study, and a total of 74 and 143 patients were evaluated. The median dialysis duration for MHD patients was 39.7 months. Table 1 presents the baseline characteristics of the patients. Incident HD patients were significantly older ( $\mathrm{p}=0.001)$ than MHD patients and used arteriovenous grafts (AVGs), as opposed to arteriovenous fistulas (AVFs), more frequently than the MHD patients $(p=0.01)$. Preoperative Doppler ultrasound was typically performed prior to long-term vascular access to identify suitable vessels for AVF

Table 1. Baseline characteristics of all patients

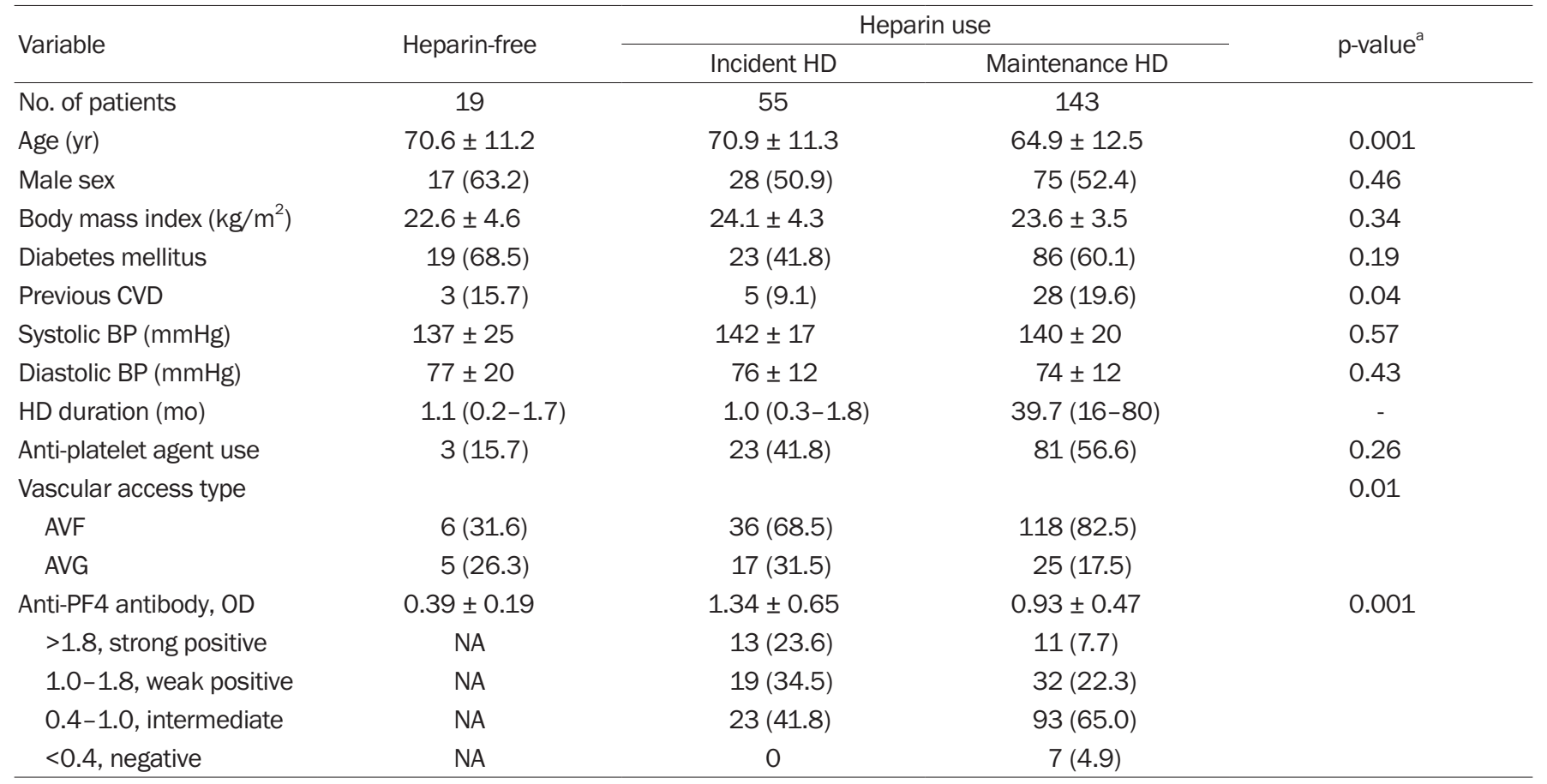

Number (\%) only, mean \pm standard deviation, or median (interquartile range).

AVF, arteriovenous fistula; AVG, arteriovenous graft; BP, blood pressure; CVD, cardiovascular disease; HD, hemodialysis; NA, not applicable; OD, optical density; PF4, platelet factor 4.

${ }^{a} \mathrm{p}$-value for comparison between incident HD and maintenance HD. 
placement; if no suitable vessels were present, an AVG was placed. Other baseline comorbidities were comparable between the two dialysis groups.

We divided the patients into three groups; incident HD patients with heparin free, incident HD patients with heparin use, and MHD patients. We then compared the anti-heparin/PF4 antibody levels among the three groups. As expected, anti-heparin/PF4 antibodies were rarely detected in non-heparinized HD patients (mean OD, $0.39 \pm 0.11$ ). Notably, the mean anti-heparin/PF4 antibody OD value was significantly higher in incident HD patients compared to MHD patients, even though both HD patient groups were regularly exposed to heparin ( $1.34 \pm 0.65$ vs. $0.93 \pm 0.47$, p $<0.001$ ) (Fig. 1). Applying the cutoff OD value of 1.8 (i.e., strongly positive), the prevalence of clinically significant levels of anti-heparin/PF4 antibodies was $23.6 \%$ in incident HD patients (13 of 55) and 7.7\% in MHD patients (11 of 143). The prevalence of heparin-induced antibodies in the MHD group was comparable to previously reported data.

Focusing on the incident HD group, we examined the relationships between clinical and biochemical parameters and clinically significant anti-heparin/PF4 antibody levels (Table 2). Patients with antibody OD values of $>1.8$ showed an increased tendency toward higher systolic and diastolic blood pressures than the control group. Notably,

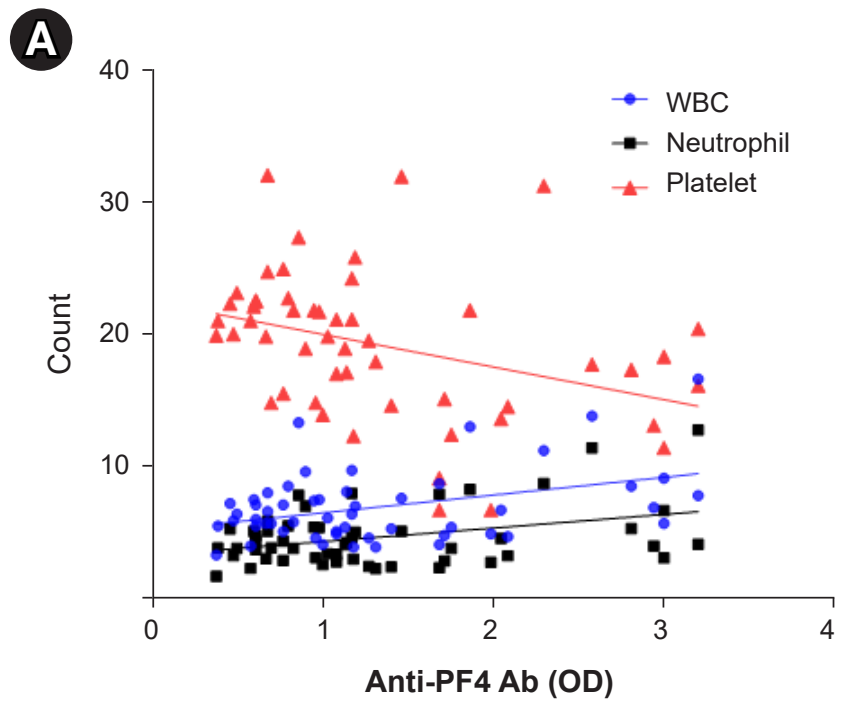

patients with strongly positive anti-heparin/PF4 antibody levels had significantly higher WBC and neutrophil counts compared to the controls, even though all cell counts were within normal ranges (Fig. 2A, Table 3). However, the number of platelets was slightly lower in patients with clinically

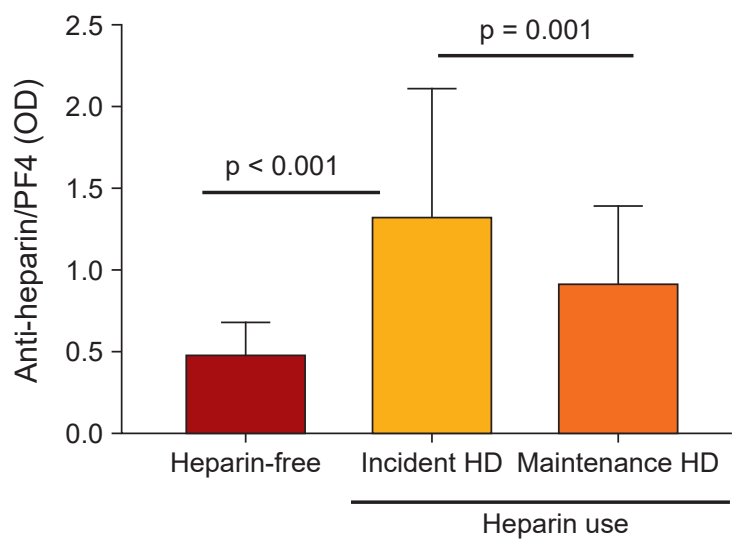

Figure 1. Differences in anti-heparin/PF4 antibody levels among groups. In patients without heparin use (heparin-free), the antibody level was very low (negative control). In patients with heparin use, the level was significantly higher in incident HD patients compared to maintenance HD patients.

$\mathrm{HD}$, hemodialysis; PF4, platelet factor 4.
B

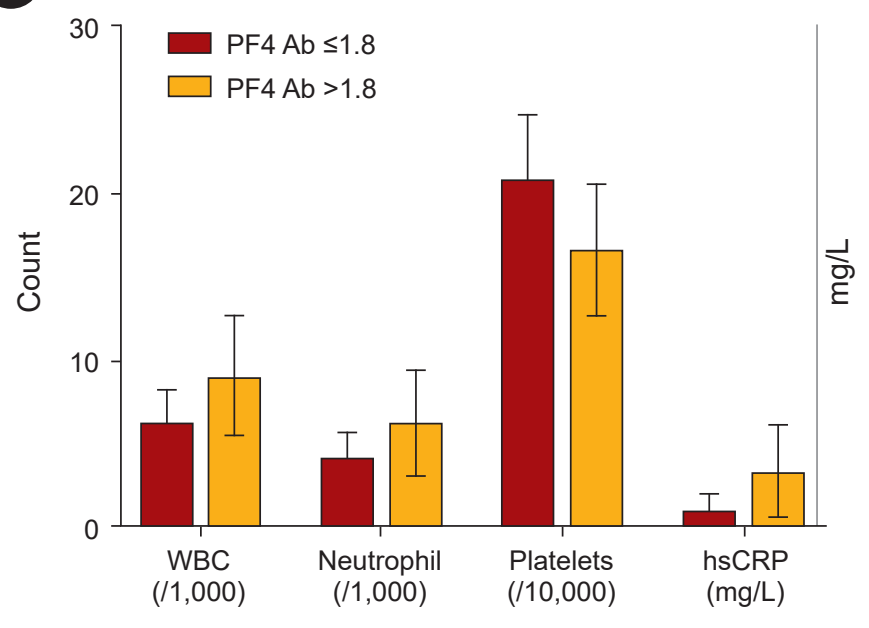

Figure 2. The relationship between anti-heparin/PF4 Ab levels and immune cell counts. (A, B) The Ab level had a positive correlation with WBC and neutrophil counts but had a negative relationship with platelet count. Also, the levels of hs-CRP, a serum inflammatory marker, were significantly higher in patients with strongly positive Ab levels (B).

$\mathrm{Ab}$, antibody; hs-CRP, high-sensitivity C-reactive protein; OD, optical density; PF4, platelet factor 4; WBC, white blood cell. 
Table 2. Comparison of biochemical data and vascular outcomes according to PF4 antibody level

\begin{tabular}{|c|c|c|c|}
\hline \multirow{2}{*}{ Variable } & \multicolumn{2}{|c|}{ Incident HD with heparin use $(n=55)$} & \multirow{2}{*}{ p-value } \\
\hline & $\mathrm{OD} \leq 1.8(\mathrm{n}=42)$ & $O D>1.8(n=13)$ & \\
\hline Age (yr) & $70.8 \pm 11.2$ & $69.1 \pm 11.7$ & 0.93 \\
\hline Male sex & $21(50.0)$ & $5(38.5)$ & 0.22 \\
\hline Diabetes mellitus & $24(57.1)$ & $6(46.1)$ & 0.47 \\
\hline Previous CVD & $3(7.1)$ & $2(15.3)$ & 0.30 \\
\hline Systolic BP (mmHg) & $139.6 \pm 19.0$ & $148.5 \pm 16.4$ & 0.08 \\
\hline Diastolic BP (mmHg) & $74.0 \pm 13.0$ & $80.1 \pm 9.9$ & 0.09 \\
\hline Vascular access type & & & 0.27 \\
\hline AVF & $29(69.0)$ & $8(61.5)$ & \\
\hline AVG & $13(31.0)$ & $5(38.5)$ & \\
\hline \multicolumn{4}{|l|}{ Biochemical parameter } \\
\hline WBC count & $6,427 \pm 1,960$ & $9,205 \pm 3,580$ & 0.001 \\
\hline Hemoglobin (g/dL) & $10.5 \pm 1.3$ & $10.9 \pm 1.3$ & 0.71 \\
\hline Neutrophil count & $4,350 \pm 1,509$ & $6,435 \pm 3,116$ & 0.004 \\
\hline Neutrophil/lymphocyte ratio & $4.0 \pm 2.9$ & $4.3 \pm 2.6$ & 0.77 \\
\hline Platelet $\left(10^{3} / \mathrm{mL}\right)$ & $200 \pm 40$ & $168 \pm 70$ & 0.07 \\
\hline$<100 \times 10^{6} / \mathrm{mL}$ & $2(4.7)$ & $2(15.4)$ & \\
\hline $\mathrm{BUN}(\mathrm{mg} / \mathrm{dL})$ & $57.6 \pm 17.6$ & $54.4 \pm 22.6$ & 0.58 \\
\hline Creatinine (mg/dL) & $6.8 \pm 1.9$ & $6.3 \pm 1.9$ & 0.46 \\
\hline Albumin $(g / d L)$ & $3.6 \pm 0.6$ & $3.6 \pm 0.3$ & 0.90 \\
\hline Total cholesterol (mg/dL) & $154.1 \pm 29.3$ & $148.6 \pm 36.9$ & 0.56 \\
\hline hs-CRP & $1.10 \pm 1.04$ & $3.50 \pm 2.70$ & 0.006 \\
\hline \multicolumn{4}{|l|}{ Vascular outcome } \\
\hline \multicolumn{4}{|l|}{ Primary outcome } \\
\hline Loss of primary patency in $6 \mathrm{mo}$ & $9(21.4)$ & $7(53.8)$ & 0.03 \\
\hline Abandonment in 6 mo & $0(0)$ & $4(30.7)$ & \\
\hline First intervention in 6 mo & $9(21.4)$ & $6(46.2)$ & \\
\hline \multicolumn{4}{|l|}{ Secondary outcome } \\
\hline Time to first needling (day) & $55.5 \pm 16.7$ & $72.7 \pm 34.3$ & 0.03 \\
\hline Time to first intervention (mo) & $5.2 \pm 4.0$ & $13.4 \pm 6.9$ & 0.07 \\
\hline First intervention in 3 mo & $6(14.3)$ & $6(46.2)$ & 0.02 \\
\hline \multicolumn{4}{|l|}{ Time to reintervention } \\
\hline Re-PTA in 3 mo & $4(9.5)$ & $5(38.4)$ & 0.02 \\
\hline Abandonment during follow-up & $3(7.1)$ & 6 (46.1) & 0.003 \\
\hline
\end{tabular}

Data are expressed as mean \pm standard deviation or number (\%).

AVG, arteriovenous graft; BP, blood pressure; BUN, blood urea nitrogen; CVD, cardiovascular disease; HD, hemodialysis; hs-CRP, high-sensitivity C-reactive protein; OD, optical density; PF4, platelet factor 4; PTA, percutaneous transluminal angioplasty; WBC, white blood cell.

Table 3. Correlation analysis of Anti-PF4 antibody with nucleosome level and blood cell variables

\begin{tabular}{|c|c|c|c|c|c|c|c|c|}
\hline \multirow{2}{*}{ Variable } & \multicolumn{2}{|c|}{ WBC } & \multicolumn{2}{|c|}{ Neutrophil } & \multicolumn{2}{|c|}{ Platelet } & \multicolumn{2}{|c|}{ Nucleosome leve } \\
\hline & $r$ & $p$ & $r$ & $p$ & $r$ & $p$ & $r$ & $p$ \\
\hline Anti-PF4 antibody & 0.397 & 0.003 & 0.37 & 0.006 & -0.37 & 0.005 & 0.31 & 0.03 \\
\hline WBC & - & & 0.96 & $<0.001$ & 0.31 & 0.02 & 0.2 & 0.08 \\
\hline Neutrophil count & - & - & - & - & 0.29 & 0.03 & 0.35 & 0.04 \\
\hline
\end{tabular}

PF4, platelet factor 4; WBC, white blood cell. 
significant anti-heparin/PF4 antibody levels. The frequency of thrombocytopenia (defined as a platelet count $<1 \times 10^{8} /$ $\mathrm{mL}$ ) was higher in the patients with high anti-heparin/PF4 antibody levels; however, because of the low number of cases, this finding was not statistically significant. Levels of the inflammatory marker hs-CRP were also statistically higher in patients with high anti-heparin/PF4 antibody levels (Fig. 2B). The other metabolic parameters were similar between patients with and without high anti-heparin/PF4 antibody levels. No relationship was found between the type of vascular access (AVF or AVG) and the anti-heparin/PF4 antibody positivity rate.

\section{Vascular outcomes of patients positive for anti-heparin/ platelet factor 4 antibodies}

Anti-heparin/PF4 antibodies are associated with platelet activation and endothelial cell damage; therefore, we conducted thorough follow-up examinations to evaluate the HD vascular outcomes of all patients. Vascular complications are largely due to thrombosis and stenosis following endothelial damage and inflammation. Our data showed that patients with strong positive anti-heparin/PF4 antibodies had significantly higher rates of primary functional patency loss within 6 months of the first HD compared to the control group (53.8\% vs. $21.4 \%, \mathrm{p}=0.03$ ) (Fig. 3A). Relative risk calculations revealed that patients with an anti-heparin/PF4 antibody OD of $>1.8$ were 4.28 times more likely to experience primary functional patency failure compared to those with an anti-heparin/PF4 antibody OD of $\leq 1.8$. One-third of the patients exhibiting strong positive antibodies experienced vascular access abandonment within 6 months, whereas no control group patients lost vascular access within the same period. In addition, around twice as many patients in the strongly positive anti-heparin/PF4 antibody group underwent vascular interventions within 6 months of vascular access creation ( $46.2 \%$ vs. $21.4 \%$ ).

We then examined the relationships between the presence of clinically significant anti-heparin/PF4 antibody levels and the times of first cannulation and first intervention. There were no cases of primary maturation failure up to 3 months after the creation. However, the time taken for successful cannulation in three consecutive HD sessions was significantly longer in the strongly positive anti-heparin/PF4 antibody group compared to the control group; this was likely due to the higher vascular intervention rate in the strong positive group compared to the controls $(72.7 \pm 34$.3 days vs. $55.5 \pm 16.7$ days, $p=0.03$ ). Supporting this, patients who had clinically significant anti-heparin/PF4 antibody levels experienced earlier vascular intervention compared to control patients (Fig. 3A). The mean times until the first intervention were 5.2 and 13.4 months in the strong positive and control
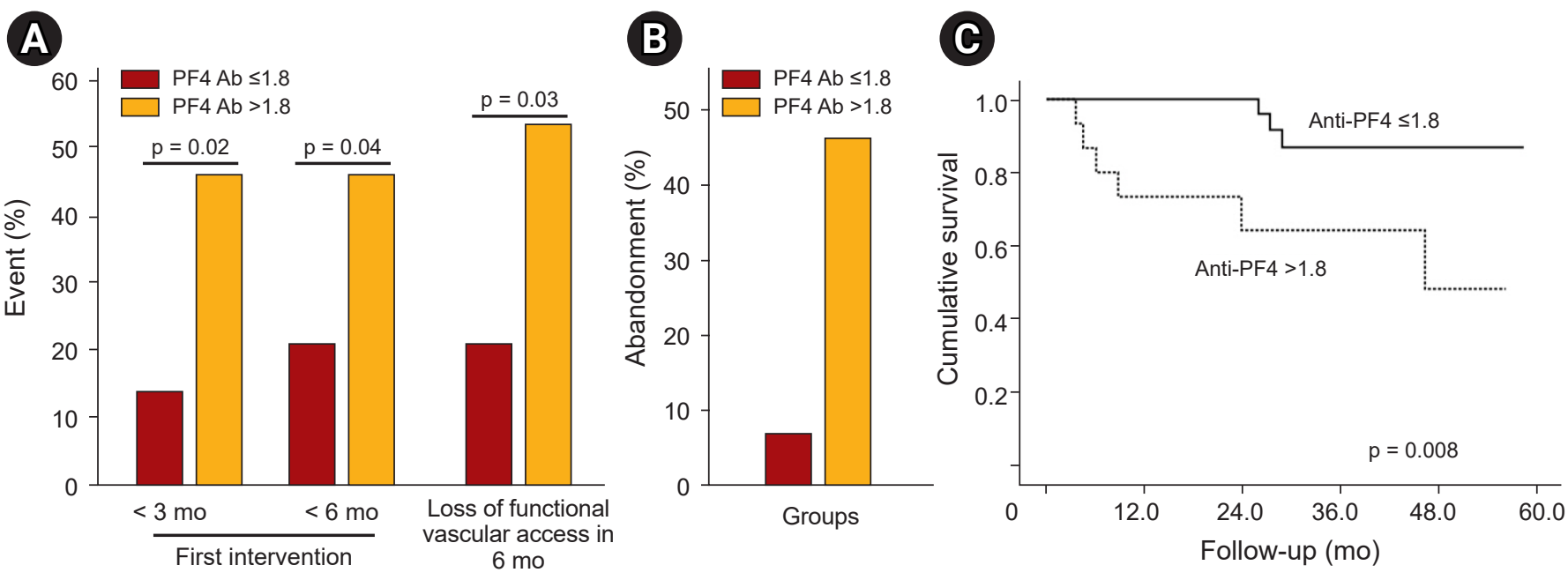

Figure 3. Vascular outcomes according to strong positivity for anti-heparin/PF4 Abs. (A) Patients with anti-heparin/PF4 Abs underwent more frequent and earlier vascular interventions compared to the control group. Therefore, they had a much higher incidence of vascular access failure. $(B, C)$ The abandonment risk was also significantly higher in patients with Abs.

Ab, antibody; PF4, platelet factor 4. 
groups, respectively. Furthermore, about half of the patients in the strong positive group underwent repeated interventions within 3 months of the initial intervention. Clinically, patients requiring frequent vascular intervention due to recurrent access obstruction could be easily identified. Taken together, our data suggested that platelet activation triggered by anti-heparin/PF4 antibody formation may be associated with early and repeated intravascular thrombotic occlusion of HD vascular access.

Additionally, we compared the rates of access abandonment between the strongly positive anti-heparin/PF4 antibody group and the control group (Fig. 3B, C). During follow-up examinations conducted $28.8 \pm 14.1$ months after this study, there were nine cases of abandonment (six and three in the strong positive and control groups, respectively). According to the Cox proportional HR, patients who were strongly positive for anti-heparin/PF4 antibodies had a 7.29fold higher risk of vascular access abandonment (95\% CI, 1.79-25.65 in univariate analysis). Even after adjusting for age, sex, diabetes, vascular type, and hs-CRP, the presence of clinically significant anti-heparin/PF4 antibodies increased the abandonment risk 7.32-fold (95\% CI, 1.31-40.88) (Table 4).

\section{Relationship of serum nucleosome level with anti-heparin/ platelet factor 4 positivity}

Due to the close association between strong anti-heparin/PF4 antibody positivity and high WBC and neutrophil counts in peripheral blood, we evaluated the relationship between anti-heparin/PF4 antibody and serum nucleosome levels. Consistent with our previous findings, the serum nucleosome levels varied significantly among the HD patients.

Table 4. Cox proportional HRs for predicting abandonment of vascular access

\begin{tabular}{|c|c|c|}
\hline Variable & $\mathrm{HR}(95 \% \mathrm{Cl})$ & p-value \\
\hline \multicolumn{3}{|l|}{ Univariate analysis } \\
\hline Anti-PF4/heparin antibody, >1.8 & $7.29(1.79-25.65)$ & 0.004 \\
\hline \multicolumn{3}{|l|}{ Multivariate analysis } \\
\hline Age, per 1 yr & $1.14(0.96-1.34)$ & 0.13 \\
\hline Diabetes mellitus, presence & $1.06(0.09-2.27)$ & 0.34 \\
\hline Vascular access type (AVG) & $3.10(0.66-14.46)$ & 0.15 \\
\hline Anti-PF4/heparin antibody, >1.8 & $7.32(1.31-40.88)$ & 0.02 \\
\hline
\end{tabular}

AVG, arteriovenous graft; $\mathrm{Cl}$, confidence interval; hs-CRP, high-sensitivity C-reactive protein; HR, hazard ratio; PF4, platelet factor 4.
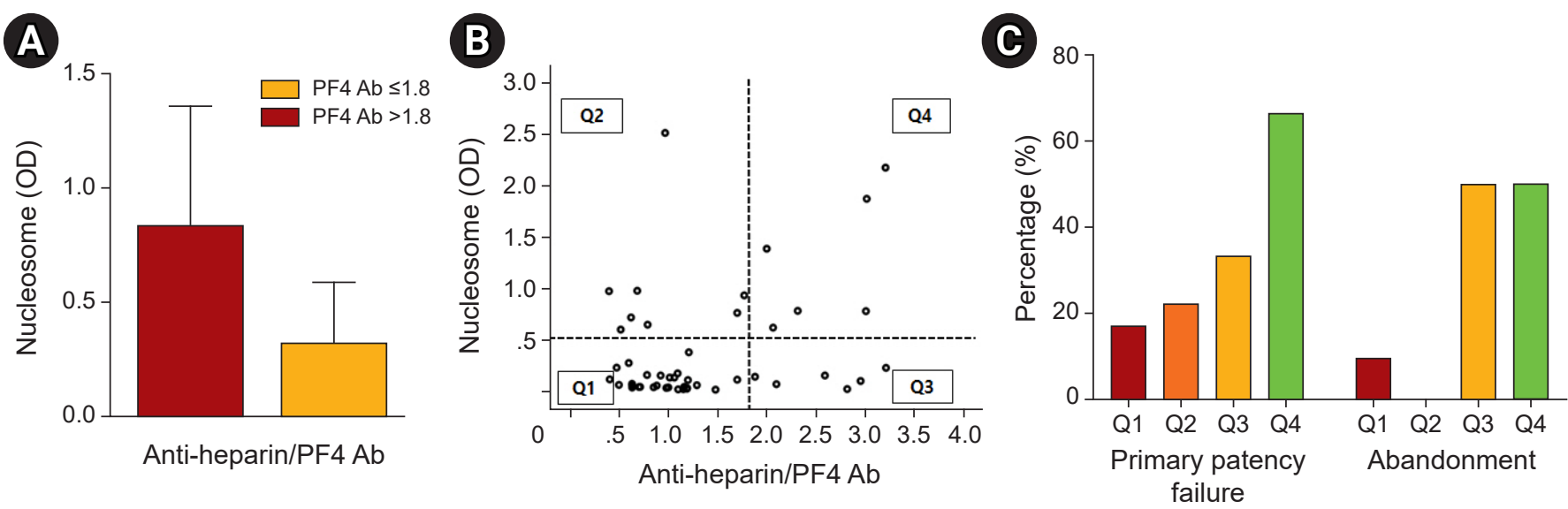

Figure 4. The relationship between anti-heparin/PF4 strong positivity and serum nucleosome level. (A) Patients with strongly positive anti-heparin/PF4 Abs had significantly higher levels of nucleosomes. (B, C) When divided into four groups according to the nucleosome highest quartile and an $\mathrm{OD}$ level of 1.8 , the highest rates of primary patency loss were observed in patients with an $\mathrm{Ab}$ OD $>1.8$ and nucleosome OD > 0.52 (Q4).

Ab, antibody; OD, optical density; PF4, platelet factor 4. 
The median serum nucleosome OD value was 0.16 , and the cutoff value for the highest quartile (Q4) was 0.52 . Serum nucleosome levels exhibited a close relationship with anti-heparin/PF4 antibody levels $(r=0.309 ; \mathrm{p}=0.03)$ (Table 3). As shown in Fig. 4A, the median nucleosome OD value was significantly higher in the strongly antibody-positive group compared to the control group ( 0.72 vs. $0.32, p=0.04$ ). We then divided the total population into four groups according to anti-heparin/PF4 antibody OD values ( $>$ or $\leq 1.8$ ) and nucleosome OD values ( $>$ or $\leq$ the Q4 cutoff value of 0.52 ) and compared the risk of primary patency loss and vascular access abandonment among the four groups (Fig. 4B, C). The highest rates of primary patency loss were observed in patients with an antibody OD of $>1.8$ and nucleosome OD of $>0.52$. Even in patients with clinically significant anti-heparin/PF4 antibody levels, the risk significantly increased with higher nucleosome levels.

\section{Discussion}

In this study, we investigated the potential impact of anti-heparin/PF4 antibodies on HD vascular access outcomes and their relationship with NET levels. There were several strengths to our study, the first being that we measured anti-heparin/PF4 antibody levels in both incident HD and MHD patients, which enabled direct comparison of the two groups. Given our results, we suggest that timing is an important factor when testing for anti-heparin/PF4 antibodies. Another strength of our study was that we only focused on vascular access. The causes of cardiovascular and cerebrovascular events in HD patients are complex and multifactorial; although the causes are similarly complex for vascular outcomes, the main pathogenesis of vascular complications is thrombotic occlusion with stenosis. Furthermore, we measured serum nucleosome levels alongside performance of the anti-heparin/PF4 antibody test since neutrophil dysregulation and increased NET formation have been reported to contribute to thrombosis in uremic conditions. Platelet-neutrophil interactions could be key in the formation of vascular access clots.

According to previous HIT studies, anti-heparin/PF4 antibody positivity rates were within the range of $2.3 \%$ to $10.3 \%$ in HD patients. The presence of HIT antibodies was not found to be associated with adverse clinical outcomes; however, previous studies only examined MHD patients, without considering incident HD patients $[3,8,9,11,12,28]$. There are multiple HIT phases, including the acute phase, subacute phase, and several recovery phases. MHD patients may fall within the subclinical or subacute HIT phase. Moreover, MHD patients may harbor clinically silent or insignificant anti-heparin/PF4 antibodies that do not cause thrombocytopenia or thrombosis. Therefore, we hypothesized that the prognostic significance of HIT antibodies should be determined in incident HD patients who have recently been exposed to heparin. In this study, we compared the prevalence of anti-heparin/PF4 antibodies between incident HD patients and MHD patients and found that the frequency of HIT antibodies was much lower in MHD patients compared to incident patients (7.7\% vs. $21.7 \%)$. Supporting this, a previous study also reported that the incidence of heparin-induced antibodies was highest during the first 90 days of HD (20\%). However, by 3 months, approximately $9 \%$ of patients had antibodies [8]. One potential explanation could be that patients who had developed anti-heparin/PF4 antibodies experienced vascular complications earlier during the treatment course and had therefore already died or been hospitalized/transferred to nursing care facilities prior to this study, and thus were not included in our MHD group. Consistent with this explanation, the average age of the MHD patients was significantly lower, and AVF was the main vascular access route rather than AVG. Another explanation for this finding is that anti-heparin/PF4 antibodies may be degraded following repeated exposures to heparin; however, this issue is beyond the scope of this investigation.

In this report, we focused on the incident HD group and further evaluated the prognostic role of anti-heparin/PF4 antibodies in vascular outcomes. Notably, patients with clinically significant antibody levels had higher WBC and neutrophil counts than those without. Consistent with this finding, serum nucleosome levels were much higher in the antibody-positive group. Taken together, this suggests that the presence of anti-heparin/PF4 antibodies was accompanied by neutrophil activation and increased NET formation in vivo. The platelet counts appeared to be lower in patients with anti-heparin/PF4 antibodies; however, the difference was not statistically significant. Therefore, an increased neutrophil count with or without thrombocytopenia may be a characteristic of anti-heparin/PF4 antibody-positive patients. Furthermore, the expression level of the inflammatory marker hs-CRP was higher in the positive antibody group 
compared to the control group. The increased NET levels, positive anti-heparin/PF4 antibodies, and hyperinflammatory condition may simultaneously contribute to vascular thrombosis in vivo.

We evaluated the value of anti-heparin/PF4 antibodies for predicting functional vascular access (defined as successfully matured and used for dialysis) and the subsequent loss of primary functional patency within 6 months of its first use. Because the main objective of the study was to investigate the thrombotic occlusion associated with anti-heparin/PF4 antibodies, patients with decompensated heart failure or decompensated liver cirrhosis were specifically excluded to rule out potential inflow problems with respect to vascular access. An interval of $<3$ months between vascular access creation and first use likely indicates that any subsequent loss of primary functional patency is not due to anatomical problems or low blood flow. Our results showed that the presence of anti-heparin/PF4 antibodies was a powerful predictor of vascular access failure (odds ratio, 7.3; $\mathrm{p}=0.02$ ), even after adjustment for diabetes and inflammatory conditions. In addition, the risks of vascular intervention within 3 and 6 months were 3.1 and 6.3 times higher, respectively, in the positive anti- heparin/PF4 antibody group than in the control group. Positive HIT antibodies were linked to a higher incidence of repeated vascular intervention within 3 months of the first vascular intervention and to the abandonment of vascular access. Consistent with our data, O'shea et al. [10] reported that hypercoagulable states are common in HD patients with recurrent vascular access site thrombosis.

Another notable finding in this study was that patients with positive anti-heparin/PF4 antibodies had higher serum nucleosome levels than the control group, and the vascular outcomes were worse in patients with both higher nucleosome levels and strongly positive antibodies. Given the strong association of the anti-heparin/PF4 antibodies with serum neutrophil and nucleosome levels, it is reasonable to suggest that an interaction between the anti-heparin/PF4 antibodies and excessive NET could be a risk factor for vascular thrombosis. Supporting our data, Perdomo et al. [21] recently reported that the HIT immune complex induces NETosis by interacting with FcrRIIa on neutrophils and through neutrophil-platelet associations. They found neutrophil-platelet aggregates in the blood of patients with HIT and then demonstrated the involvement of NETosis in thrombi generated ex vivo. Furthermore, they showed that neutrophil depletion abolished thrombus formation in vivo. Similarly, another recent report showed that anti-heparin antibodies could activate neutrophils and cause them to selectively bind to injured endothelia, where they released NETs; these NETs generated immunogenic complexes, which could then induce prothrombotic conditions. Moreover, the complexes are resistant to endonuclease digestion, which leads to repeated and prolonged clot formation $[19,29]$.

A limitation of this study was that a relatively small number of patients were analyzed, all of whom were from a single center. We tried to include more patients by extending the study period, but the availability of anti-heparin/PF4 antibody commercial kits was limited due to their infrequent use in clinical practice. Therefore, the results are not representative. However, our data are nonetheless valuable; by comparing the antibody levels between MHD and incident HD patients, we discovered a relationship between anti-heparin/PF4 antibody levels and the time of heparin exposure. Relatively few studies including incident HD patients have been published. Another limitation of this study was that although we measured serum nucleosome levels, we were unable to directly visualize NETs in vivo. Furthermore, we arbitrarily determined the cutoff OD value for positive anti-heparin/PF4 antibodies (i.e., 1.8). The prevalence of anti-heparin/PF4 antibodies differs largely among existing reports, because it is influenced by the kit and the detection method used. Even for the same kit, the cutoff value differs among studies. In this study, we applied the same cutoff value used in a previous one, and also used the same ELISA kit; the positive control yielded OD values $>1.8$ [25]. Warkentin et al. [25] reported that the probability of a strongly positive result in a serotonin ELISA was about $90 \%$ when a cutoff OD value of 1.8 to 2.0 was applied.

In conclusion, the frequency of anti-heparin/PF4 antibodies was significantly higher in incident HD patients compared to MHD patients. This likely explains why previous studies involving MHD patients failed to show any significant prognostic role of anti-heparin/PF4 antibodies. In incident HD patients, we found that the presence of anti-heparin/PF4 antibodies was associated with increased NET formation and that this could be a strong predictor for subsequent vascular access complications. This provides evidence for a pathological interaction between platelet activation, increased NET formation, and endothelial damage in vivo. 


\section{Conflicts of interest}

All authors have no conflicts of interest to declare.

\section{Funding}

This research was supported by a National Research Foundation grant funded by the Korean government (2020R1A2C110138611) and the Hallym University Research Fund.

\section{Authors' contributions}

Conceptualization, Funding acquisition: JKK, SGK

Data curation: JNA, HSL

Formal analysis: JKK, SGK, JNA, HSL

Investigation: HWL, JKK, YRS, HJK

Methodology: YRS, HJK

Writing-original draft: HWL, JKK

Writing-review \& editing: YRS, HJK

All authors read and approved the final manuscript.

\section{ORCID}

Hoi Woul Lee, https://orcid.org/0000-0001-8014-129X Jung Nam An, https://orcid.org/0000-0001-5108-1005 Hyung Seok Lee, https://orcid.org/0000-0001-6380-9243 Young Rim Song, https://orcid.org/0000-0002-0416-4745 Hyung Jik Kim, https://orcid.org/0000-0002-3379-1721 Sung Gyun Kim, https://orcid.org/0000-0002-5034-0527 Jwa-Kyung Kim, https://orcid.org/0000-0002-7726-2143

\section{References}

1. Monroy-Cuadros M, Yilmaz S, Salazar-Bañuelos A, Doig C. Risk factors associated with patency loss of hemodialysis vascular access within 6 months. Clin J Am Soc Nephrol 2010;5:1787-1792.

2. Warkentin TE, Sheppard JA, Horsewood P, Simpson PJ, Moore JC, Kelton JG. Impact of the patient population on the risk for heparin-induced thrombocytopenia. Blood 2000;96:1703-1708.

3. Kato S, Takahashi K, Ayabe K, et al. Heparin-induced thrombocytopenia: analysis of risk factors in medical inpatients. $\mathrm{Br} \mathrm{J}$ Haematol 2011;154:373-377.

4. Nand S, Wong W, Yuen B, Yetter A, Schmulbach E, Gross Fisher S. Heparin-induced thrombocytopenia with thrombosis: inci- dence, analysis of risk factors, and clinical outcomes in 108 consecutive patients treated at a single institution. Am J Hematol 1997;56:12-16.

5. Rauova L, Zhai L, Kowalska MA, Arepally GM, Cines DB, Poncz M. Role of platelet surface PF4 antigenic complexes in heparin-induced thrombocytopenia pathogenesis: diagnostic and therapeutic implications. Blood 2006;107:2346-2353.

6. Arepally GM. Heparin-induced thrombocytopenia. Blood 2017;129:2864-2872.

7. Shen JI, Winkelmayer WC. Use and safety of unfractionated heparin for anticoagulation during maintenance hemodialysis. Am J Kidney Dis 2012;60:473-486.

8. Asmis LM, Segal JB, Plantinga LC, et al. Heparin-induced antibodies and cardiovascular risk in patients on dialysis. Thromb Haemost 2008;100:498-504.

9. Hutchison CA, Dasgupta I. National survey of heparin-induced thrombocytopenia in the haemodialysis population of the UK population. Nephrol Dial Transplant 2007;22:1680-1684.

10. O'shea SI, Lawson JH, Reddan D, Murphy M, Ortel TL. Hypercoagulable states and antithrombotic strategies in recurrent vascular access site thrombosis. J Vasc Surg 2003;38:541-548.

11. Zhao D, Sun X, Yao L, et al. The clinical significance and risk factors of anti-platelet factor 4/heparin antibody on maintenance hemodialysis patients: a two-year prospective follow-up. PLoS One 2013;8:e62239.

12. Pena de la Vega L, Miller RS, Benda MM, et al. Association of heparin-dependent antibodies and adverse outcomes in hemodialysis patients: a population-based study. Mayo Clin Proc 2005;80:995-1000.

13. Fathi M. Heparin-induced thrombocytopenia (HIT): Identification and treatment pathways. Glob Cardiol Sci Pract 2018;2018:15.

14. East JM, Cserti-Gazdewich CM, Granton JT. Heparin-induced thrombocytopenia in the critically ill patient. Chest 2018;154:678-690

15. Warkentin TE, Sheppard JI, Heels-Ansdell D, et al. Heparin-induced thrombocytopenia in medical surgical critical illness. Chest 2013;144:848-858.

16. Cuker A. Management of the multiple phases of heparin-induced thrombocytopenia. Thromb Haemost 2016;116:835-842.

17. Cuker A, Arepally GM, Chong BH, et al. American Society of Hematology 2018 guidelines for management of venous thromboembolism: heparin-induced thrombocytopenia. Blood $A d v$ 2018;2:3360-3392.

18. Hayes V, Johnston I, Arepally GM, et al. Endothelial antigen as- 
sembly leads to thrombotic complications in heparin-induced thrombocytopenia. J Clin Invest 2017;127:1090-1098.

19. Gollomp K, Kim M, Johnston I, et al. Neutrophil accumulation and NET release contribute to thrombosis in HIT. JCI Insight 2018;3:e99445.

20. Khairy M, Lasne D, Amelot A, et al. Polymorphonuclear leukocyte and monocyte activation induced by plasma from patients with heparin-induced thrombocytopenia in whole blood. Thromb Haemost 2004;92:1411-1419.

21. Perdomo J, Leung HH, Ahmadi Z, et al. Neutrophil activation and NETosis are the major drivers of thrombosis in heparin-induced thrombocytopenia. Nat Commun 2019;10:1322.

22. Martinod K, Wagner DD. Thrombosis: tangled up in NETs. Blood 2014;123:2768-2776.

23. Ott U, Sperschneider H. Demands on dialysis access from the nephrologists point of view. Chirurg 2012;83:775-778.

24. Mickley V. Stenosis and thrombosis in haemodialysis fistulae and grafts: the surgeon's point of view. Nephrol Dial Transplant
2004;19:309-311.

25. Warkentin TE, Sheppard JI, Moore JC, Sigouin CS, Kelton JG. Quantitative interpretation of optical density measurements using PF4-dependent enzyme-immunoassays. J Thromb Haemost 2008;6:1304-1312.

26. Kim JK, Lee HW, Joo N, et al. Prognostic role of circulating neutrophil extracellular traps levels for long-term mortality in new end-stage renal disease patients. Clin Immunol 2020;210:108263.

27. Sidawy AN, Gray R, Besarab A, et al. Recommended standards for reports dealing with arteriovenous hemodialysis accesses. $J$ Vasc Surg 2002;35:603-610.

28. O'Shea SI, Sands JJ, Nudo SA, Ortel TL. Frequency of anti-heparin-platelet factor 4 antibodies in hemodialysis patients and correlation with recurrent vascular access thrombosis. $\mathrm{Am} \mathrm{J} \mathrm{He-}$ matol 2002;69:72-73.

29. Arepally GM, Kamei S, Park KS, et al. Characterization of a murine monoclonal antibody that mimics heparin-induced thrombocytopenia antibodies. Blood 2000;95:1533-1540. 